OPEN ACCESS

Edited by:

Giuseppe Giaccone,

Cornell University, United States

Reviewed by:

Chryso Kanthou,

The University of Sheffield,

United Kingdom

Chaoming Xia,

Soochow University Medical College

(SUMC), China

*Correspondence:

Weiqing Pan

wqpan0912@aliyun.com

Specialty section:

This article was submitted to

Cancer Molecular Targets and Therapeutics,

a section of the journal

Frontiers in Oncology

Received: 12 January 2021 Accepted: 01 June 2021

Published: 16 June 2021

Citation:

Hu C, Li Y, Pan D, Wang J, Zhu L, Lin Y, Zhu S and Pan W (2021) A Schistosoma japonicum MicroRNA

Exerts Antitumor Effects Through Inhibition of Both Cell Migration and Angiogenesis by Targeting PGAM1.

Front. Oncol. 11:652395.

doi: 10.3389/fonc.2021.652395

\section{A Schistosoma japonicum MicroRNA Exerts Antitumor Effects Through Inhibition of Both Cell Migration and Angiogenesis by Targeting PGAM1}

\author{
Chao $\mathrm{Hu}^{1}$, Yuzhen $\mathrm{Li}^{1}$, Danting Pan ${ }^{1}$, Jing Wang ${ }^{1}$, Liufang Zhu ${ }^{1}$, Yu Lin ${ }^{1}$, Shanli Zhu ${ }^{1}$ \\ and Weiqing Pan ${ }^{1,2 *}$ \\ 1 Institute for Infectious Diseases and Vaccine Development, Tongji University School of Medicine, Shanghai, China, \\ 2 Department of Tropical Diseases, Naval Medical University, Shanghai, China
}

MicroRNA (miRNA) is an important regulator for gene expression. Recent studies showed that some heterogenous miRNAs derived from both parasite and plant can regulate expression of mammalian gene in a cross-species or even a cross-kingdom manner. Here, we identified a Schistosoma japonicum miRNA (designated as sja-miR-61) that is present in the hepatocyte of mice infected with the parasite. The sja-miR-61 mimics significantly inhibited the migration of both mouse and human hepatoma cells in vitro. In a xenograft animal model, significant reductions of the tumor volume and weight were observed in mice inoculated with hepatoma cells transfected with sja-miR-61 mimics compared to the controls. We found that the in vivo inhibition of tumor growth was through its antiangiogenesis activity. Mechanically, we identified the phosphoglycerate mutase 1 (PGAM1) gene as a target of sja-miR-61 and found that the sja-miR-61-mediated suppression of cell migration and anti-angiogenesis by cross-species down-regulation of PGAM1 expression. These data indicated that sja-miR-61 is a tumor suppressor miRNA that may have therapeutic potential for human cancers.

Keywords: Schistosoma japonicum, microRNA, hepatoma cell, PGAM1, cross-species regulation

\section{INTRODUCTION}

MicroRNAs (MiRNAs) is a class of highly conserved small non-coding RNAs and play critical roles in regulation of gene expression via binding to their target mRNAs (1). Studies have shown that dysregulated expression of some miRNAs are involved in the occurrence and development of a number of diseases such as cancers $(2,3)$. These miRNAs may serve as targets for therapeutic intervention $(4,5)$. Interestingly, recent studies have shown that miRNAs derived from parasites and plants can regulate the expression of mammalian target genes in a cross-species or even a crosskingdom manner, and thereby affecting the occurrence and development of some human diseases (6-9). For example, the plant-derived miR-159 suppressed the growth of breast cancer cell via cross-

Abbreviations: S. japonicum, Schistosoma japonicum; HCC, hepatocellular cancer; PGAM1, phosphoglycerate mutase 1; miRNA, microRNA; siRNA, small interfering RNA; NC, negative control; Mock, mock control. 
kingdom regulation of the human transcription factor 7 (TCF7) gene (8). Importantly, abundance of miR-159 in the serum of patients was inversely correlated with incidence and progression of breast cancer (8).

Schistosomiasis is a neglected tropical parasitic disease that affects approximately 200 million people in the world's tropical areas (10). For Schistosoma japonicum (S. japonicum) infection, numerous eggs laid by the female adult worms were trapped in both liver and intestinal wall tissues, leading to a granulomatous reaction and hepatic fibrosis. Our previous study demonstrated that $S$. japonicum eggs can secrete exosomes that contain S. japonicum miRNAs (sja-miRNAs) (11). In addition, sjamiRNAs can be detected in the infected liver hepatocytes and hepatic stellate cells, indicating that sja-miRNAs can be taken up by the host liver cells during the infection $(11,12)$. We showed that a schistosome-derived sja-miR-2162 that is present in host hepatic stellate cells of infected mice can upregulate collagens and $\alpha$-SMA production to promote hepatic fibrosis by targeting transforming growth factor beta receptor III (12). In addition, we reported two schistosome-derived miRNAs, sja-miR-3096 and sja-miR-7-5p, that were present in hepatocytes of mice infected with $S$. japonicum inhibited the growth of hepatoma cells by targeting host genes $(13,14)$. In the present study, we identified an additional S. japonicum-specific miRNA-61 (designated as sja-miR-61), which is enriched in extracellular vesicles secreted by S. japonicum (EVs) (11), is also present in hepatocytes during the S. japonicum infection. This schistosome miRNA suppressed tumor cell migration in vitro and growth of hepatoma in vivo through anti-angiogenesis by targeting PGAM1 gene.

\section{MATERIALS AND METHODS}

\section{Cell Culture}

Both the Hepa1-6 and HepG2 cell lines were grown in Dulbecco's modified Eagle's medium (DMEM, Life Technologies, USA) supplemented with $10 \%(\mathrm{v} / \mathrm{v})$ fetal bovine serum (Invitrogen, USA), $100 \mathrm{U} / \mathrm{ml}$ penicillin, and $100 \mu \mathrm{g} / \mathrm{ml}$ streptomycin (Invitrogen, USA) at $37^{\circ} \mathrm{C}$ in a $5 \% \mathrm{CO} 2$ incubator, while the HUVEC cell line was grown in Endothelial Cell Medium (ECM, ScienCell, USA), supplemented with $10 \%$ (v/v) fetal bovine serum (Invitrogen, USA), 1× Endothelial Cell Growth Supplement (ECGS, ScienCell, USA), $100 \mathrm{U} / \mathrm{ml}$ penicillin, and $100 \mu \mathrm{g} / \mathrm{ml}$ streptomycin (Invitrogen, USA) at $37^{\circ} \mathrm{C}$ in a $5 \% \mathrm{CO} 2$ incubator.

\section{Transfection of MiRNA Mimics and Small Interfering RNA}

For mimics and small interfering RNA (siRNAs) transfection, exponential growing cells were seeded in the culture plate overnight and transfected with $60 \mathrm{nM}$ sja-miR-61 mimics, siRNAs or negative control mimics (NC; a negative control mimic that has no target gene in human and mice) (Genepharma, Shanghai, China) using Lipofectamine 3000 (Invitrogen, Carlsbad, CA, USA), sja-miR-61mimics: sense 5'UGA CUA GAA AGU GCA CUC ACU U-3'; anti-sense 5'-GUG AGU GCA CUU UCU AGU CAU U-3'. Human PGAM1
siRNA-1: sense 5'-GUC CUG UCC AAG UGU AUC UTT-3'; anti-sense 5'-AGA UAC ACU UGG ACA GGA CTT-3'. Human PGAM1 siRNA-2: sense 5'-CCA CAU CUG UAG ACA UCU UTT-3'; anti-sense 5'- AAG AUG UCU ACA GAU GUG GTT3'. Human FERMT2 siRNA: sense 5'-CUG GUG GAG AAA CUC GAU GUA TT-3'; anti-sense 5'-UAC AUC GAG UUU CUC CAC CAG TT-3'. Murine Pgam1 siRNA-1: sense 5'-CCC UAG AAG GUU GGG AUC ATT-3'; anti-sense 5'-UGA UCC CAA CCU UCU AGG GTT-3'. Murine Pgam1 siRNA-2: sense 5'-CGC CUC AAU GAG CGA CAC TTT-3'; anti-sense 5'-AGU GUC GCU CAU UGA GGC GTT-3’. Murine Fermt2 siRNA: sense 5'-UUG GUG GAA AAA CUC GAU GUC TT-3'; antisense 5'-GAC AUC GAG UUU UUC CAC CAA TT-3'. NC mimics or NC siRNA: sense 5'-UUC UCC GAA CGU GUC ACG UTT-3'; anti-sense 5'-ACG UGA CAC GUU CGG AGA ATT-3'. For plasmids transfection, exponential growing cells were seeded in the culture plate overnight and transfected with plasmids using Lipofectamine 3000 (Invitrogen, Carlsbad, CA, USA). The cells were incubated for the period of the indicated time, and then subjected to further analysis as described below.

\section{In Vitro Migration Assay}

Cell migration was measured by both the Transwell migration and wound healing assay. For Transwell migration assay, Hepa16 or HepG2 cells $\left(2 \times 10^{5}\right)$ and HUVEC $\left(5 \times 10^{4}\right)$ were seeded in a 6-well plate overnight, then cells were transfected with sja-miR61 mimics, NC mimics or Mock control (transfection reagents only), respectively. And $24 \mathrm{~h}$ later, cells were digested and $2 \times 10^{4}$ Hepa1- 6 or HepG2 cells, $1 \times 10^{4}$ HUVEC cells were transferred into the upper chamber in $100 \mu \mathrm{L}$ of serum-free medium, and $500 \mu \mathrm{L}$ compete medium with $10 \%$ (v/v) fetal bovine serum was added to the lower chamber, three replicates per group. And $24 \mathrm{~h}$ later, the upper chambers were fixed in methanol for 30 minutes, followed by staining in crystal violet for 15 minutes. After stained, cells on the upper surface of the membrane were wiped out lightly by using the small cotton ball, cells on the lower surface of the membrane were photographed and counted under a light microscope in five fields. For wound healing assay, cells $\left(3 \times 10^{5}\right)$ cells were seeded in a 6-well plate overnight and then transfected with miRNA mimics as above description. Once confluent, cells were scratched in a straight line using a $200 \mu \mathrm{L}$ sterile pipette tip. Then, suspended cells were washed off with PBS and cultured in DMEM with $1 \%(\mathrm{v} / \mathrm{v})$ fetal bovine serum culture. The scratched area was photographed at 0 and $48 \mathrm{~h}$, respectively. The relative area of migration formula $=\mathrm{A} / \mathrm{B}$ (Where $\mathrm{A}$ is the area of migrated cells in experimental group after $48 \mathrm{~h}$; where B is the area of migrated cells in control group after $48 \mathrm{~h}$ ). The area of migrated cells is evaluated by the Image 1.42q (ImageJ software, Way Rasband, National Institutes of Health, USA).

\section{Luciferase Reporter Assay}

The 3' untranslated region (UTR) wild-type (WT) and mutant (MT) of PGAM1 were amplificated from mouse genome or human genome, and then cloned into the Sac I/Xba I site of pmirGLO Dual-Luciferase miRNA Target Expression Vector (Promega, USA), including human pmirGLO-PGAM1-WT and 
mouse pmirGLO-Pgam1-WT (containing a wild type binding site in the 3' UTR of PGAM1), human pmirGLO-PGAM1-MT and mouse pmirGLO-Pgam1-MT (containing a mutant type binding site), and these luciferase reporters were simultaneously transfected with sja-miR-61 mimics or NC mimics in Hepa1-6 or HepG2 cells, respectively. Dual-luciferase reporter assay system (Promega, USA) was used to measure the activity of the reporter gene according to the manufacturer's instructions, and the firefly luciferase activity was normalized to renilla luciferase activity.

\section{Mice and S. japonicum Infections}

All the animal experiments were performed in accordance with the Guide for the Care and Use of Laboratory Animals of the National Institutes of Health, and approved by the Internal Review Board of Tongji University School of Medicine. All the animal surgeries were undertaken under sodium pentobarbital anesthesia. Thirty-six male C57BL/6J mice (6 weeks old, 18-20 g) were purchased from experimental animal center of the Second Military Medical University and housed under specific pathogen-free conditions. Cercariae of S. japonicum were provided by National Institute of Parasitic Disease, Chinese Center for Disease Control and Prevention (CDC). Mice were percutaneously infected with cercariae of S. japonicum, 50 cercariae for collection of infected hepatocytes and 100 cercariae for collection of early stage parasites (3 mice per group). For collection of parasites, the S. japonicum were isolated from the portal system and mesenteric veins of infected mice at 7, 14 and 42 days post-infection (dpi). In addition, at 42 days, female and male adult worms were manually separated. All the freshly isolated parasites were washed with PBS and immediately used for extraction of total RNA or frozen at $-80^{\circ} \mathrm{C}$ until used.

\section{PGAM1 Overexpression Plasmid Construction}

The coding sequence of murine Pgam 1 gene and human PGAM1 gene were amplified in mouse cDNA or human cDNA, respectively, and cloned into pcDNA3.1(+) vector (Invitrogen, Carlsbad, CA, USA), and the empty pcDNA3.1 (+) vector was used as a control. The primer sequences amplified the PGAM1 coding sequence are listed in Table S1.

\section{Hepatocellular Carcinoma Xenografts}

Six male athymic nude mice (6 weeks old) were housed and manipulated according to the protocols approved by the Shanghai Medical Experimental Animal Care Commission. Hepa1-6 cells or HepG2 cells were transfected with sja-miR-61 mimics or NC mimics at a final concentration of $60 \mathrm{nM}$, respectively. And $24 \mathrm{~h}$ later, $1 \times 10^{6}$ cells in $100 \mu \mathrm{L}$ PBS after treated with sja-miR-61 mimics or NC mimics were implanted subcutaneously to per scapula of the nude mice, respectively. The tumor volume was measured every two days after injection. After the last measure, the nude mice were sacrificed and the tumors were separated to evaluate their weight and volume. The tumor volume was determined using the formula: $0.5 \times \mathrm{L} \times \mathrm{S}^{2}$, where $\mathrm{L}$ or $\mathrm{S}$ are the longest or shortest diameter of tumor, respectively. The level of sja-miR-61 mimics transfected into the hepatoma cells were detected by quantitative real-time polymerase chain reaction (qRT-PCR), the expression of Ki67 and CD34 in the tumor was measured by immunohistochemistry as described under this section.

\section{Western Blot Analysis}

The Western blot analysis was performed as described previously (15). Briefly, the cell and tissue sample lysates, extracted by cell lysis buffer (Beyotime, China) and the concentration was measured by Enhanced BCA Protein Assay Kit (Beyotime, China) according to the manufacturer's instructions. About $20 \mu \mathrm{g}$ of protein was separated by $12 \%$ SDS-PAGE and transferred to a nitrocellulose membrane, respectively. Then the membrane was blocked with $5 \%$ bovine serum albumin (BSA) in Tris-buffered saline with Tween (TBST) for $2 \mathrm{~h}$ at room temperature, followed by incubated overnight with primary antibodies against PGAM1 (1:500 dilution, Proteintech, China), GAPDH (1:1000 dilution, Beyotime, China). After incubating with the first antibody, the membrane was washed three times with TBST, and then incubated with the relevant secondary antibodies (1:6000 dilution, Promega, USA) for $1 \mathrm{~h}$ at room temperature and followed by three times washes, then visualized by using the ECL reagent (GE Healthcare, UK), the protein bands were subsequently measured using the ImageQuant LAS 4000mini (GE Healthcare, USA) and grayscale analysis using the ImageJ 1.42q (ImageJ software, Way Rasband, National Institutes of Health, USA).

\section{Immunohistochemistry}

To determine Ki67, CD34 expression in xenograft tumor tissues from the athymic nude mice, immunohistochemistry (IHC) was performed as described previously (16), Antibody against Ki67, CD34 was used (1:50 dilution). Three mice from each group were used for IHC, and three sections of each tumor were used for analyzing the expression of Ki67 and CD34. For quantification, all IHC photographs were analyzed by using the ImageJ $1.42 \mathrm{q}$ (ImageJ software, Way Rasband, National Institutes of Health, USA), following the ImageJ User Guide. Differences in Ki67 and CD34 staining were evaluated by assessing the relative area of positive zone.

\section{Statistical Analysis}

All experiments were performed in triplicate and the results were presented as mean \pm standard deviation (mean \pm SD). All data were analyzed by one-way ANOVA using the software GraphPad Prism 5.0 (GraphPad Software, Inc. La Jolla, CA, USA). A value of $P<0.05$ was considered statistically significant.

\section{RESULTS}

\section{Presence of Sja-miR-61 in Host Hepatocytes of Infected Mice}

We first evaluated the presence of sja-miR-61 in the liver cells of infected mice. The sja-miR-61 is a Schistosoma-specific miRNA, 
which allowed us to design the specific primers for detection of this schistosome sja-miR-61 in the hepatocytes of mice infected with S. japonicum (Table S1). To exclude any contamination from parasite RNA, we carefully prepared the samples of liver cells from the infected mice and showed that a parasite reference gene $(N A D H)$ with high expression was not detectable in the samples (Figure S1A). Then we detected the various time-point samples for presence of sja-miR-61 by qRT-PCR. As shown in Figure S1B, the sja-miR-61 was detected at a higher level in the liver cells from early-infected mice (i.e., days 9 and 14 post infection) and the late-infected mice (day 42 post infection) compared with the rest time-point samples. The presence of sjamiR-61 was further verified by PCR as shown in the agarose gel (Figure S1C). Furthermore, the PCR product showed identical sequence of sja-miR-61 by cloning and sequencing (Figure S1D). These data indicated that sja-miR-61 is present in the host hepatocytes during S. japonicum infection.

\section{Inhibition of Migration of Hepatoma Cells by Sja-miR-61}

To investigate the anti-tumor effects of sja-miR-61 on hepatoma cells in vitro, both mouse hepatoma cell lines (Hepa1-6) and human hepatoma cell lines (HepG2) were transfected with the sja-miR-61 mimics, respectively. We showed that the transfected sja-miR-61 mimics were present in the both cell lines (Figure 1A), and consequently, significantly inhibited the cell migration of the hepatoma cell lines detected by the transwell migration assay (Figure 1B) and the wound healing assay (Figures S2A, B) compared with the NC or Mock group. However, we did not observe any inhibitory effect of the sjamiR-61 on the cell cycle and no effect on apoptosis of the hepatoma cell lines (Figures S3A, B). These data indicated that sja-miR-61 inhibited the cell migration of both mouse and human hepatoma cells in vitro.

\section{Antitumor Effects of Sja-miR-61 on Hepatoma In Vivo}

To evaluate potential effect of sja-miR-61 on hepatoma in vivo, both Hepa1-6 and HepG2 cells were transfected with the sjamiR-61 mimics or NC mimics and then injected subcutaneously into the athymic nude mice respectively to generate subcutaneous tumors. The tumor volume and weight were evaluated at days $2,4,6$, and 8 post injection. As shown in Figures 2A-C, significant reductions in both the tumor volume and weight were observed in the mice inoculated with Hepa1-6 cells transfected with sja-miR-61 mimics compared to those with NC miRNAs. In addition, the similar results were obtained in human hepatoma cell line of HepG2 (Figures 2D-F). These data suggested that sja-miR-61 inhibited tumor growth in vivo.

\section{Target Gene of Sja-miR-61}

To identify the target gene of sja-miR-61, we used the online software miRDB (17), RNA hybrid (18) and MR-microT (19) to search for putative targets of this miRNA. We found that the seed

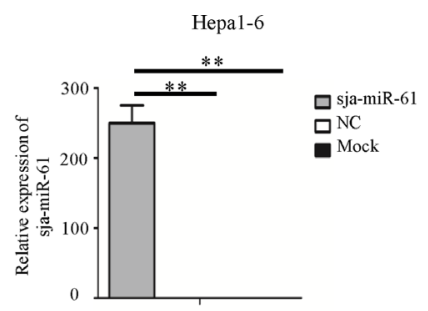

B

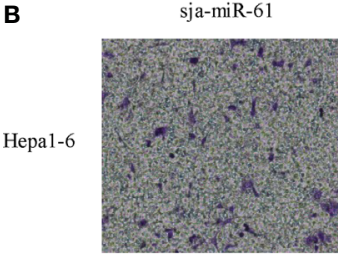

sja-miR-61

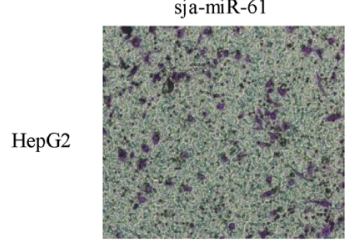

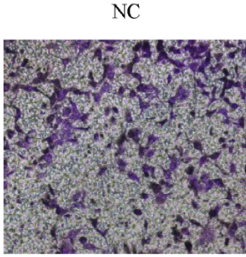

NC

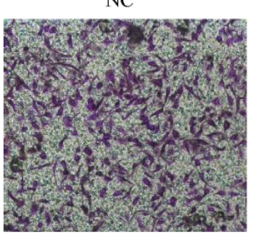

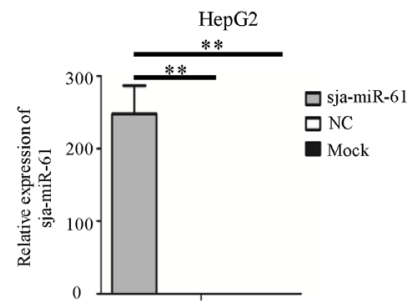
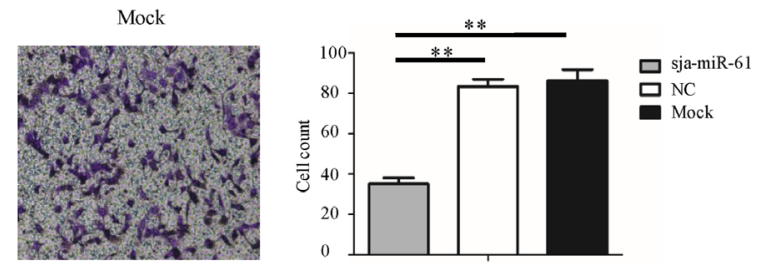

Mock
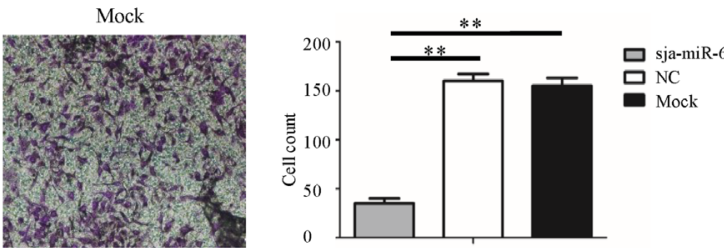

ㅁock

FIGURE 1 | Sja-miR-61-mediated inhibition of migration of hepatoma cells in vitro. Hepa1-6 and HepG2 cells were transfected with sja-miR-61 mimics and NC (negative control). mimics, respectively, and $48 \mathrm{~h}$ later, the sja-miR-61 was detected by qRT-PCR (A). Cell migration was evaluated using transwell inserts without matrigel coating (B). Data are presented as the mean $\pm \mathrm{SD}, \mathrm{n}=3,{ }^{\star \star} p<0.01$. 
A

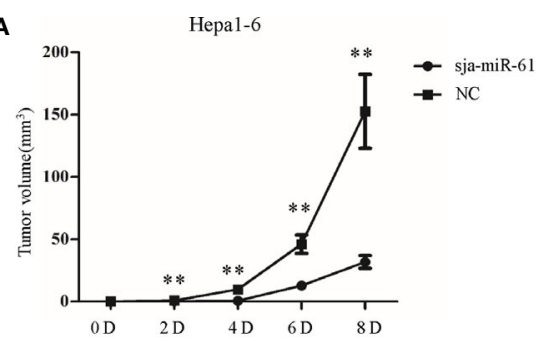

B

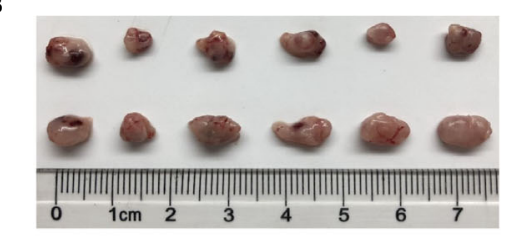

C

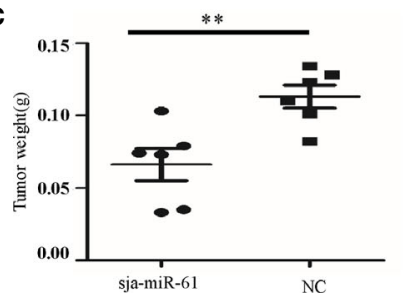

D

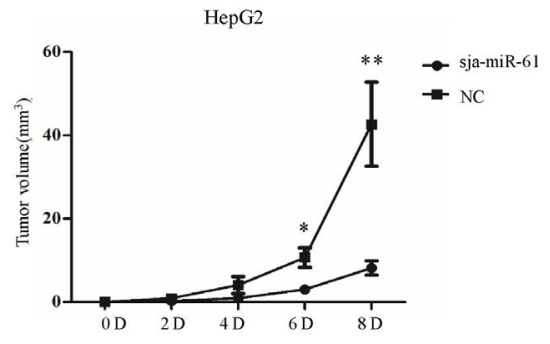

E

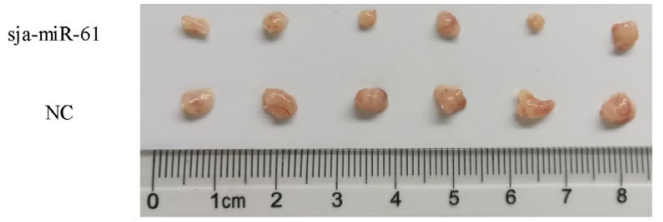

$\mathbf{F}$

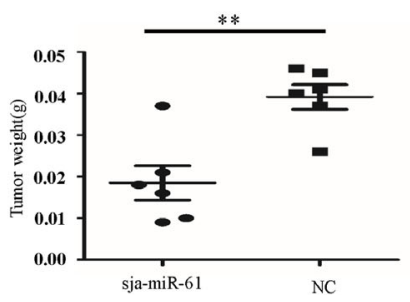

FIGURE 2 | Sja-miR-61-mediated suppression of hepatoma growth in vivo. Hepa1-6 and HepG2 cells were transfected with sja-miR-61 mimics or NC mimics, respectively, and then the sja-miR-61-transfected cells $\left(1 \times 10^{6}\right)$ were injected subcutaneously to the left scapula of athymic nude mice, and the NC-transfected cells to the right scapula $(n=6)$, respectively. Tumor volumes were measured at days $2,4,6$, and 8 after injection. At day 8 , the mice were sacrificed and tumors were separated to measure their weight and volume, (A-C) for Hepa1-6 cells, (D-F) for HepG2 cells. Data are presented as the mean $\pm \mathrm{SD}, \mathrm{n}=6,{ }^{*} p<0.05,{ }^{* *} p<0.01$.

sequence of sja-miR-61 perfectly binds to the 3' untranslated region (UTR) of the phosphoglycerate mutase 1 (PGAM1) gene of both murine and human. In addition, the PGAM1 gene was reported as an oncogene during tumorigenesis in humans (20-22).

To verify the PGAM1 gene as the target of sja-miR-61, we constructed several plasmids that expressing the luciferase reporter, in which the firefly luciferase gene is fused to the 3' UTR of PGAM1 gene of human (pmirGLO-PGAM1-WT) and murine (pmirGLO-Pgam1-WT), as well as their mutants (pmirGLO-PGAM1-MT and pmirGLO-Pgam1-MT, respectively) where the seven nucleotides of the binding site were mutated (Figure 3A). Both Hepa1-6 and HepG2 cells were simultaneously transfected with the relevant plasmids and sjamiR-61 mimics or NC mimics. As shown in Figure 3B, a significant reduction of the luciferase activity was detected in the Hepa1-6 cells transfected with the pmirGLO-Pgam1-WT but not with the pmirGLO-Pgam1-MT. Similar results were obtained with the human cell line of HepG2 (Figure 3B). These results suggested that sja-miR-61 directly bind to the 3' UTR of the PGAM1 gene, but not its mutant, to down-regulate the expression of luciferase reporter gene.

We then evaluated the expression of the PGAM1 protein in both Hepa1-6 and HepG2 cells by Western blotting after transfected with sja-miR-61 mimics. The results showed that sja-miR-61 significantly down-regulated the levels of PGAM1 in hepatoma cells compared to the NC or Mock controls (Figures 3C, D).

\section{Sja-miR-61-Mediated Inhibition of the Cell Migration by Targeting PGAM1 In Vitro}

To investigate whether sja-miR-61 inhibits the migration of hepatoma cells by targeting PGAM1, both Hepa1-6 and HepG2 cells were transiently transfected with the PGAM1 siRNAs, NC or Mock controls. We found that both murine Pgam1 siRNA and human PGAM1 siRNA obviously reduced the PGAM1 expression in Hepa1-6 cells and HepG2 cells, as measured by qRT-PCR and Western blotting, respectively (Figure 4A). Importantly, similar to the findings in the sjamiR-61 mimics-transfected cells, the siRNAs treatment led to inhibition of migration of both Hepa1-6 and HepG2 cells compared to the NC- or Mock-treatments (Figure 4B).

We next transfected both Hepa1-6 and HepG2 cells with the constructs expressing the murine Pgam1 or human PGAM1 genes respectively that lack its 3' UTR so that sja-miR-61 should not affect the expression of the transfected PGAM1 gene. As shown in Figures 5A, B, the sja-miR-61 significantly reduced the expression of endogenous $P G A M 1$ gene, but not the transfected gene in both cell lines, as the expression of PGAM1 returned to the original level when transfected with the PGAM1expressing constructs compared to the vector control. Importantly, restoration of PGAM1 level led to abolishment of 
A

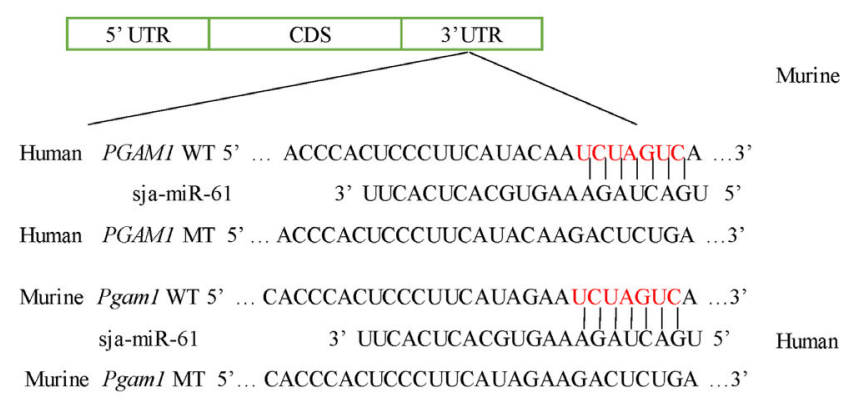

C

Hepa1-6

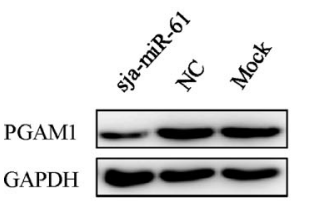

B
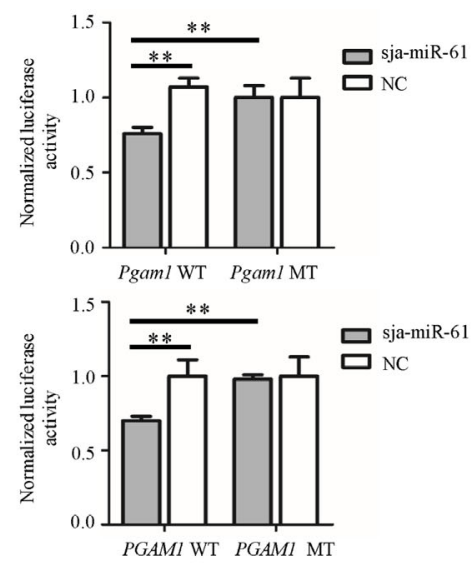

D

HepG2

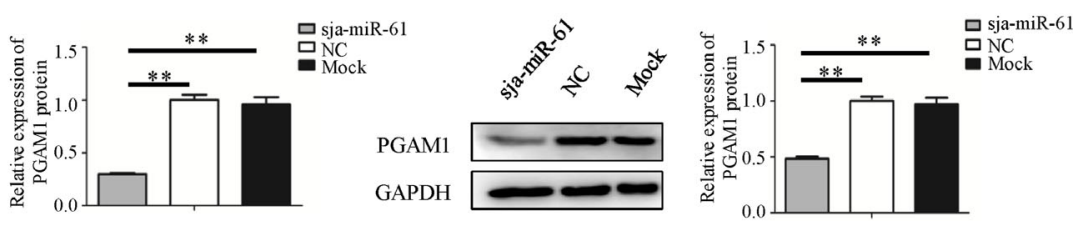

FIGURE 3 | PGAM1 as target of sja-miR-61. (A) A schematic diagram representing the wild-type or mutant 3' untranslated region (UTR) targeting sites of murine Pgam1 and human PGAM1 genes. (B) A dual-luciferase reporter assay was used to measure the activity of the reporter gene, and the firefly luciferase activity was normalized to renilla luciferase activity. (C, D) The protein levels of murine PGAM1 (C) and human PGAM1 (D) were measured using Western blotting in the hepatoma cells transfected with sja-miR-61 mimics or NC mimics, respectively. Data are presented as the mean $\pm \mathrm{SD}, \mathrm{n}=3,{ }^{\star *} p<0.01$.

the sja-miR-61-mediated inhibition of the migration of the hepatoma cell in both cell lines (Figures 5C, D). These results indicated that the inhibitory effects of the sja-miR-61 on migration of the hepatoma cells by down-regulation of the PGAM1 expression.

\section{Sja-miR-61-Mediated Suppression of the Hepatoma Growth In Vivo Through Anti- Angiogenesis by Targeting PGAM1 Gene}

As described above, sja-miR-61 has no inhibitory effect on growth of both Hepa1-6 and HepG2 cells in vitro (Figures S3A, B), but significantly suppressed growth of tumor cells in vivo (Figures 2A-F). To explore the potential molecular mechanism by which sja-miR-61 inhibited in vivo growth of hepatoma, we detected the expression levels of both Ki67 and CD34 using immunohistochemistry (IHC). CD34 is an angiogenesis marker and expressed in certain type of cells including capillary endothelial cells. We showed no change in the expression of $\mathrm{Ki67}$, indicating no impact on the cell proliferation, similar to the data from in vitro experiments. However, the expression of CD34 was significantly decreased in tumor tissues of both Hepa1-6 and HepG2 cells transfected with the sja-miR-61 mimics compared with the NC control (Figures 6A-D), suggesting the inhibitory effect of sja-miR-61 on angiogenesis in the tumor.

We next investigate if the sja-miR-61 mediates inhibition of angiogenesis by targeting $P G A M 1$ gene. Migration of vascular endothelial cells is important factor that affect angiogenesis (23). Thus, we used the human umbilical vein endothelial cells (HUVEC) for the assays of the migration. The HUVEC cells were transfected with the sja-miR-61 mimics and PGAM1 siRNA, respectively. As shown in Figures 7A, B, both sjamiR-61 mimics and PGAM1 siRNA significantly reduced the PGAM1 expression in the HUVEC cells detected by qRT-PCR. Importantly, the transwell migration showed that transfection of the HUVEC cells with either sja-miR-61 mimics or PGAM1 siRNA led to significantly inhibition of the cell migration compared with the NC control, respectively (Figures 7C). These data suggested that the inhibitory effects of the sjamiR-61 on the migration of HUVEC could be through downregulating PGAM1 expression.

To understand how the sja-miR-61 mimics enter vascular endothelial cells in vivo, we first detected the presence of sjamiR-61 in the tumors. We found that the sja-miR-61 was still detectable in the tumors on day 8 after injection of the transfected hepatoma cells with the miRNA, although the level was much lower than that on day 0 (Figures 7D, E). We speculated that the transfected sja-miR-61 mimics in the hepatoma cells can be secreted to the extracellular, and then ingested by vascular endothelial cells. To verify this, a conditioned medium (CM) was prepared by cultivation of the HepG2 cells transfected with sja-miR-61 mimics, and then the CM was used for in vitro culture of HUVEC cells. We detected and showed the presence of the sja-miR-61 inside the HUVEC 
A
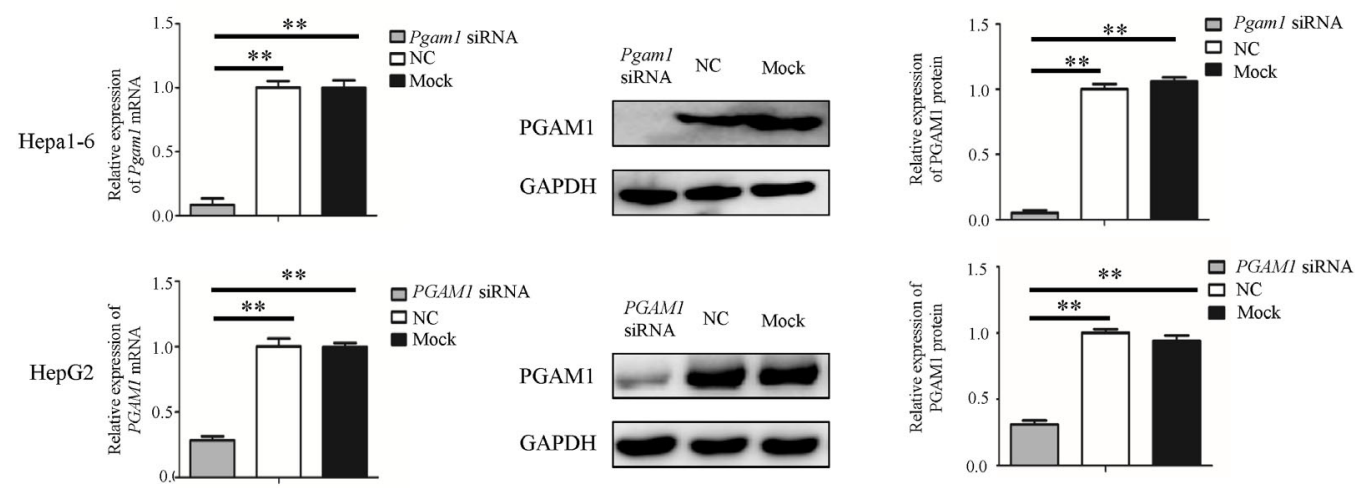

B
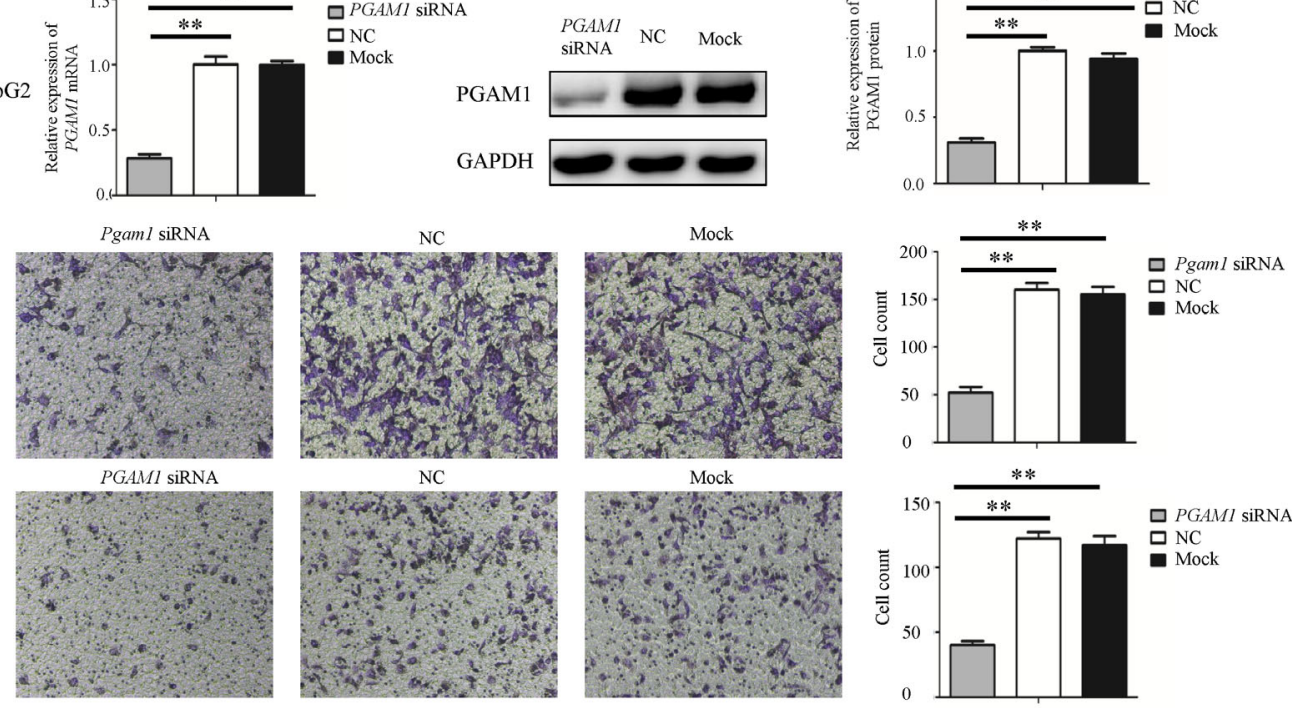

FIGURE 4 | Knockdown of PGAM1 inhibits cell migration of hepa1-6 and HepG2 cells in vitro. (A, B) Hepa1-6 and HepG2 cells were transfected with PGAM1 siRNA and negative control (NC) siRNA, respectively, and $48 \mathrm{~h}$ later, the expression of PGAM1 was determined using qRT-PCR and Western blotting (A). Cell migration was evaluated using transwell inserts without matrigel coating (B). Data are presented as the mean $\pm \mathrm{SD}, \mathrm{n}=3,{ }^{\star \star} p<0.01$.

cells (Figure 7F), suggesting that the sja-miR-61 mimic can be secreted from the transfected hepatoma cells to the medium and then ingested by the endothelial cells.

\section{DISCUSSION}

Accumulated evidences have demonstrated the anti-tumor effects of certain miRNAs through suppression of tumor cell growth, migration and invasion, including those miRNAs derived from plants and parasites. In this study, we showed that the schistosome miRNA, sja-miR-61, is present in host hepatocytes during schistosome infection, has evident inhibition of migration of hepatoma cells in vitro. Importantly, this schistosome miRNA exert a notable anti-angiogenesis activity as the expression of the angiogenesis marker (CD34) was significantly decreased in tumor tissues. The inhibition of angiogenesis activity could be through suppression of migration of the vascular endothelial cells which we demonstrated in vitro experiments. As to question about the entering of the sja-miR-61 mimics into vascular endothelial cells in vivo, we showed that the transfected hepatoma cells with the sja-miR-61 mimics could secrete the miRNA to the culture medium and ingested by the endothelial cells in an in vitro model, and that the sja-miR-61 mimics were detectable in the tumors till the $8^{\text {th }}$ day, which imply that in vivo the transfected tumor cells may secrete the sja-miR-61 mimics to the extracellular and then enter the vascular endothelial cells to exert anti-angiogenesis. In addition, the analysis of molecular mechanism revealed that sja-miR-61 exerts the inhibitory effects on both cell migration and angiogenesis by targeting the PGAM1 gene. Thus, the present data indicated that the schistosome sjamiR-61 is a tumor suppressor miRNA that may have therapeutic potential for human cancers.

Infection with certain parasites have been reported to be associated with cancers, such as Clonorchis sinensis and Opisthorchis viverrine $(24,25)$. It was also documented that Schistosoma haematobium infection is associated with bladder cancer $(24,25)$. For S. japonicum infection, a potential association with colorectal cancer was reported (26), but it is less evident for the association between S. japonicum infection and HCC. Previous studies revealed that chronic inflammation was involved in the tumorigenesis (27). As to S. japonicum infection, the parasite eggs induce severe chronic inflammation and fibrosis, which should be risk factors for HCC (28). Thus, we speculated that the eggs of $S$. japonicum trapped in the liver may play a dual role in the occurrence and development of HCC, i.e. carcinogenic and anti-cancer activities, as reported in the infection with protozoan Trypanosoma cruzi (29). The present study demonstrated that the sja-miR-61 derived from $S$. 

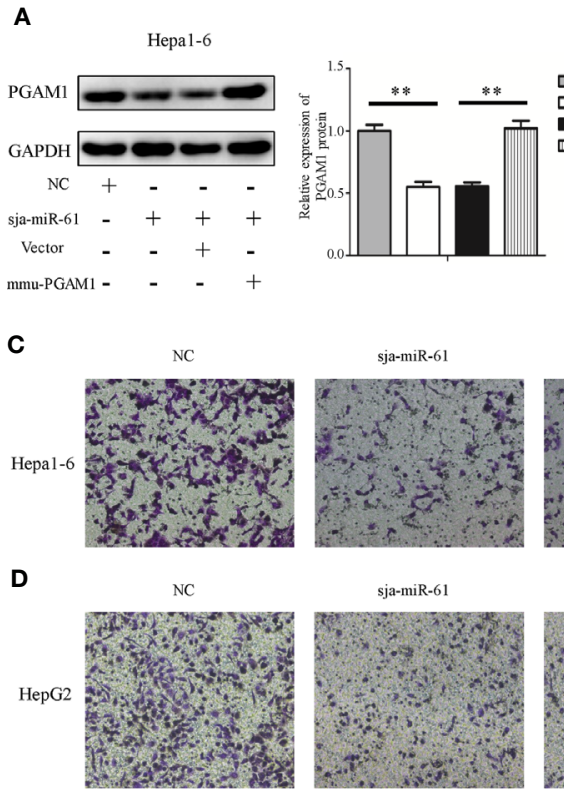
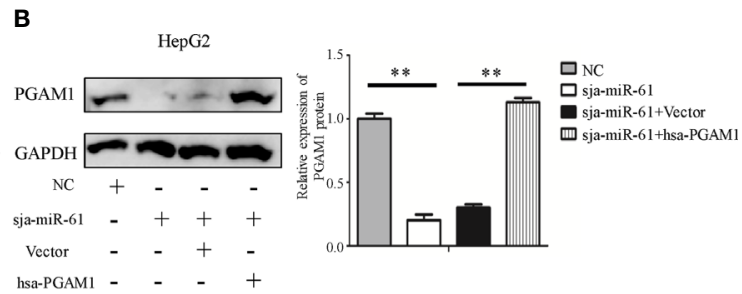

hsa-PGAM1 - - -
sja-miR-61+ Vector

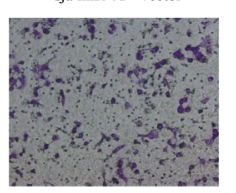

sja-miR-61+ Vector

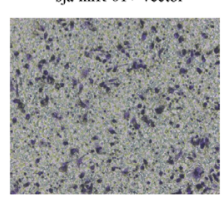

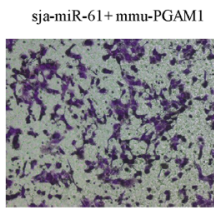

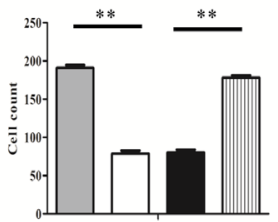

믈
․ja-miR-61

sja-miR-61+Vector

四 sja-miR-61+mmu-PGAMI
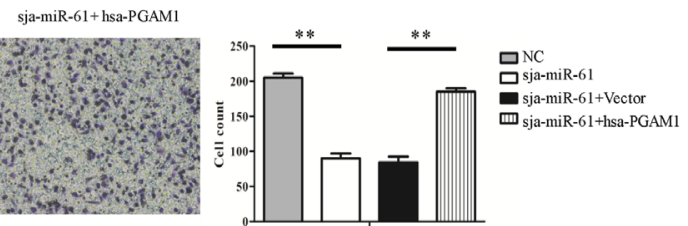

FIGURE 5 | Effect of restoration of PGAM1 expression on the sja-miR-61-mediated effects in vitro. (A-D) Cells were transfected with NC mimics, sja-miR-61 mimics, NC mimics + pcDNA3.1(+) vector, sja-miR-61 mimics + pcDNA3.1(+)-PGAM1, respectively, and $48 \mathrm{~h}$ later, the expression of PGAM1 was determined using Western blotting (A, B). Cell migration was evaluated using transwell inserts without matrigel coating (C, D). Mean $\pm \mathrm{SD}, \mathrm{n}=3,{ }^{\star \star} \mathrm{p}<0.01$.

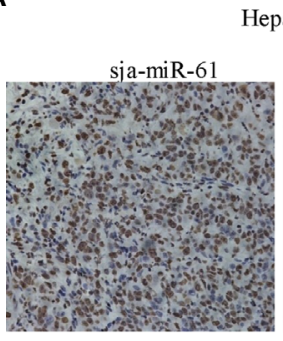

Hepal-6
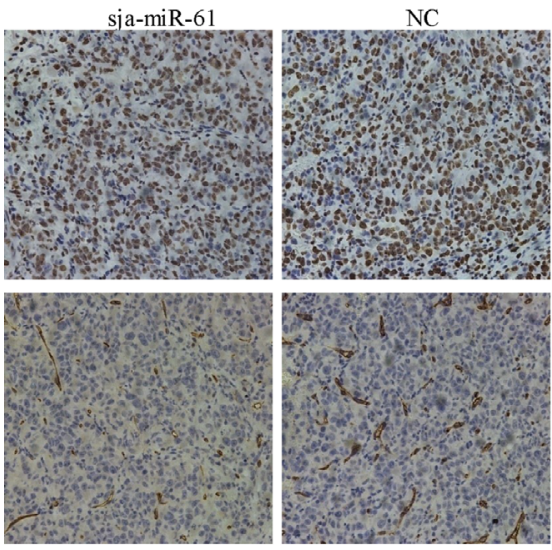

B
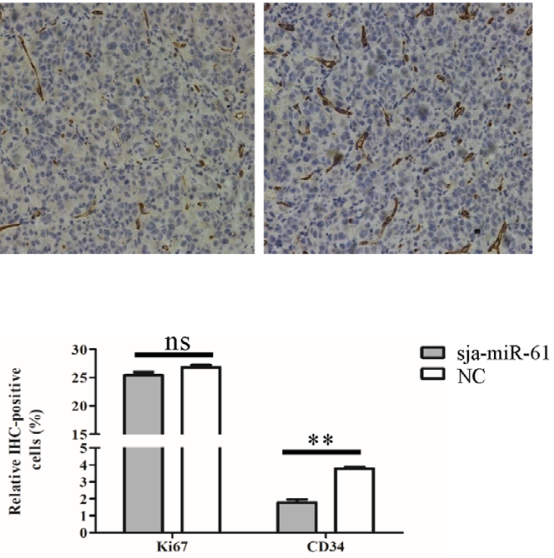

C

HepG2

Ki67
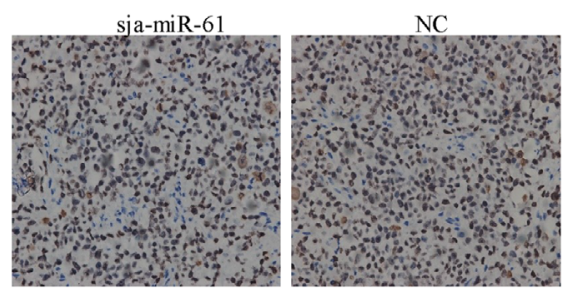

CD34
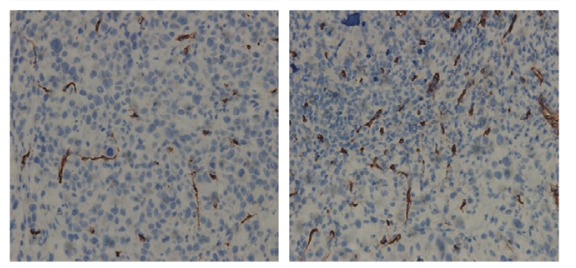

D

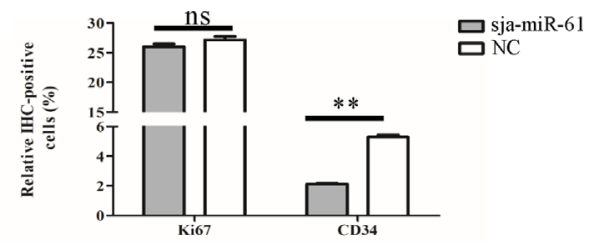

FIGURE 6 | Detection of the expression of both Ki67 and CD34 in tumors. (A-D) The expression levels of both Ki67 and CD34 in tumors were determined using immunohistochemistry, (A, B) for Hepa1-6 cells, (C, D) for HepG2 cells. Data are presented as the mean $\pm S D, n=6,{ }^{\star \star} p<0.01, n s$ indicates no significant. 

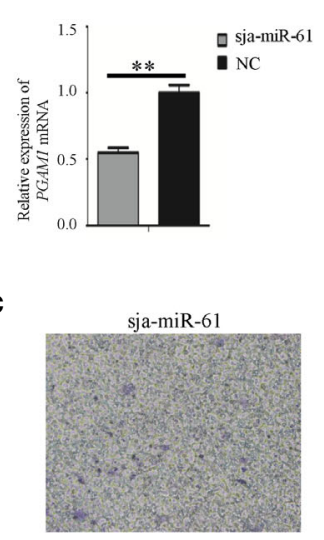

D

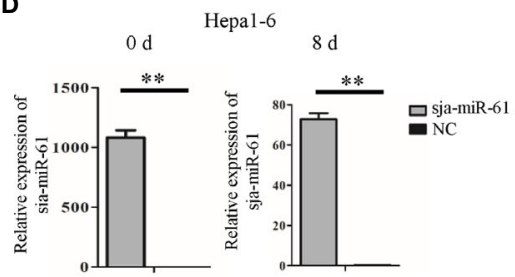

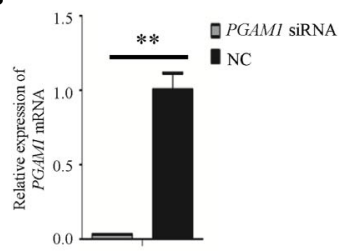

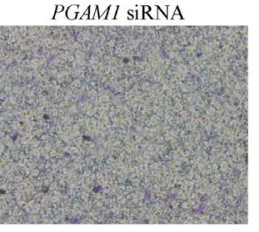

E

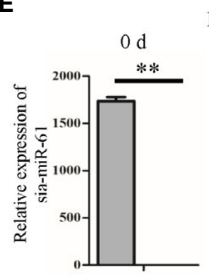

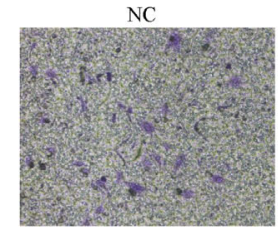

HepG2
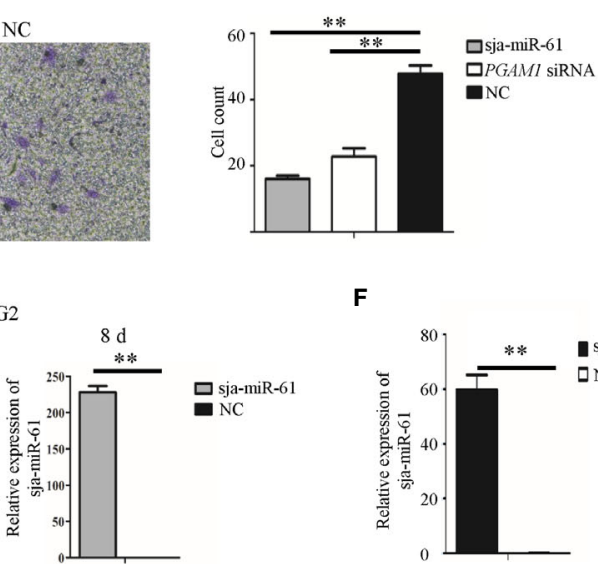

F

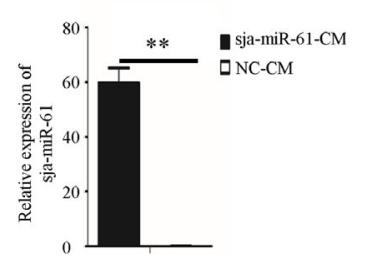

FIGURE 7 | Sja-miR-61-mediated inhibition of migration and tube formation of HUVEC cells by down-regulating PGAM1 in vitro. (A, B) HUVEC cells were transfected with sja-miR-61 mimics and NC mimics (A), PGAM1 siRNA and NC siRNA (B), respectively, and 48 h later, the expression of PGAM1 was determined using qRT-PCR. Cell migration was evaluated using transwell inserts without matrigel coating (C). (D, E) The sja-miR-61 mimics within the transfected cells were measured by qRT-PCR at day 0 (i.e. before inoculation) and 8 post inoculation, using U6 as the internal control, (D) for Hepa1-6 cells, (E) for HepG2 cells. (F) The sja-miR-61 mimics were detected by qRT-PCR in HUVEC cells cultured with conditioned medium (CM) derived from the cultivation of HepG2 cells transfected with sja-miR-61 mimics or NC mimics. Mean $\pm \mathrm{SD}, \mathrm{n}=3$, ${ }^{* *} \mathrm{p}<0.01$.

japonicum had a notable anti-tumor activity. Thus, both the presence of this miRNA, together with the other reported schistosome miRNAs such as sja-miR-3096 (13) and sjamiR-7-5p (14), in host hepatocytes and their antitumor effects on human hepatoma cells may suggest the schistosome miRNAmediated anti-tumor effects during schistosome infection.

To identify the target gene of the parasite sja-miR-61, we first used three online software to search for its potential target genes. We found 5 potential target genes (SKP2, SMO, SDCBP, FERMT2 and PGAM1) that were consistently predicted by the three softwares and involved in tumor-related signaling pathway. The luciferase reporter assay showed the genes of SMO, SDCBP, FERMT2 and PGAM1 as potential targets of the sja-miR-61 (Figure 3; Figures S4A-F; Figures S5A, B). However, the transfection experiments with siRNA or miRNA mimics excluded the SMO and SDCBP as the targets (Figures S4G, H). For both PGAM1 and FERMT2 gene, we observed that sja-miR61 down-regulated their expression, which led to the inhibition of the hepatoma cell migration in vitro, similar to that in the cell with their siRNA (Figure 3 and Figure S5), but the PGAM1mdediated inhibition degree was higher than the FERMT2mediated inhibition in terms of their expression levels and the cell migration. Thus, we focused on the PGAM1 gene as the major target of the sja-miR-61 for next experiments, including the evaluation of the inhibitory effect on migration of vascular endothelial cells, and the restoration of PGAM1 expression. We demonstrated that the restoration of PGAM1 expression rescued the sja-miR-61-mediated anti-tumor effects.

PGAM1 is an important enzyme in glycolysis, which catalyzes the conversion of 3-phosphoglycerate (3-PG) to 2 -phosphoglycerate (2-PG) (21). Several studies reported that PGAM1 is overexpressed in various cancers, including hepatocellular carcinoma (30), lung cancer (31), breast cancer (32), prostate cancer (22) and renal clear cell carcinoma (33). PGAM1 is characterized as an oncogene, and is involved in modulation of the cell proliferation, migration, invasion (20-22). In the present study, our data suggested that PGAM1 be involved in regulation of angiogenesis. Tumor angiogenesis is essential for tumor growth and metastases. We demonstrated that the sjamiR-61 inhibited migration of vascular endothelial cells in vitro through targeting PGAM1 gene, which consequently affected angiogenesis in vivo.

Tumor microenvironment (TME), a complex ecosystem comprising of tumor cells, immune cells, and stromal populations including vascular cells and fibroblasts $(34,35)$. Our data showed that vascular endothelial cells in tumors could take up sja-miR-61 secreted by tumor cells, which in turn affects the migration of vascular endothelial cells, and thereby hinders tumor growth. However, it is undeniable that the sja-miR-61 treated hepatoma cells might affect their own 
growth through autocrine, and might also affect other stromal cells through paracrine, which in turn affects the growth of tumor cells. Thus, our study has several limitations: first, it is not clear if the sja-miR-61 treated hepatoma cells can affect the growth of vascular endothelial cells, stroma cells and tumor cells through autocrine and paracrine interactions; Second, our data showed that in vitro sja-miR-61 has an inhibitory effect on the migration of hepatoma cells and vascular endothelial cells. However, the effect in vivo has not been explored yet, including the effect of sja-miR-61 on tumor metastasis; Third, the effect of sja-miR-61 on clinical tumors is not examined as it is extremely difficult to obtain clinical samples with schistosomiasis.

In conclusion, these data imply that sja-miR-61 might strengthen resistance of host to cancer during schistosome infection, and the discovery and development of such heterogenous anti-tumor miRNAs may provide a novel approach for human cancer therapeutic intervention.

\section{DATA AVAILABILITY STATEMENT}

The original contributions presented in the study are included in the article/Supplementary Material. Further inquiries can be directed to the corresponding author.

\section{ETHICS STATEMENT}

All the animal experiments were performed in accordance with the Guide for the Care and Use of Laboratory Animals of the

\section{REFERENCES}

1. Bartel DP. MicroRNAs: Genomics, Biogenesis, Mechanism, and Function. Cell (2004) 116(2):281-97. doi: 10.1016/S0092-8674(04)00045-5

2. Calin GA, Croce CM. MicroRNA Signatures in Human Cancers. Nat Rev Cancer (2006) 6(11):857-66. doi: 10.1038/nrc1997

3. Berindan-Neagoe I, Monroig PD, Pasculli B, Calin GA. MicroRNAome Genome: A Treasure for Cancer Diagnosis and Therapy. Ca Cancer J Clin (2014) 64(5):311-36. doi: 10.3322/caac.21244

4. Fang YX, Xue JL, Shen Q, Chen JZ, Tian L. MicroRNA-7 Inhibits Tumor Growth and Metastasis by Targeting the Phosphoinositide 3-kinase/Akt Pathway in Hepatocellular Carcinoma. Hepatology (2012) 55(6):1852-62. doi: 10.1002/hep.25576

5. Hatziapostolou M, Polytarchou C, Aggelidou E, Drakaki A, Poultsides GA, Jaeger SA, et al. An HNF4 Alpha-miRNA Inflammatory Feedback Circuit Regulates Hepatocellular Oncogenesis. Cell (2011) 147(6):1233-47. doi: 10.1016/j.cell.2011.10.043

6. Zhang L, Hou DX, Chen X, Li DH, Zhu LY, Zhang YJ, et al. Exogenous Plant MIR168a Specifically Targets Mammalian LDLRAP1: Evidence of Cross-Kingdom Regulation by microRNA. Cell Res (2012) 22(1):107-26. doi: 10.1038/cr.2011.158

7. Zhou Z, Li XH, Liu JX, Dong L, Chen Q, Liu JL, et al. Honeysuckle-Encoded Atypical microRNA2911 Directly Targets Influenza A Viruses. Cell Res (2015) 25(1):39-49. doi: 10.1038/cr.2014.130

8. Chin AR, Fong MY, Somlo G, Wu J, Swiderski P, Wu XW, et al. CrossKingdom Inhibition of Breast Cancer Growth by Plant miR159. Cell Res (2016) 26(2):217-28. doi: 10.1038/cr.2016.13

9. Buck AH, Coakley G, Simbari F, McSorley HJ, Quintana JF, Le Bihan T, et al. Exosomes Secreted by Nematode Parasites Transfer Small RNAs to Mammalian
National Institutes of Health, and approved by the Internal Review Board of Tongji University School of Medicine.

\section{AUTHOR CONTRIBUTIONS}

$\mathrm{CH}$ and WP conceived and designed the study. CH, YLi, DP, JW, LZ, YLin and SZ performed the experiments. $\mathrm{CH}, \mathrm{YLi}, \mathrm{DP}$ and WP analyzed the data. $\mathrm{CH}$ and WP wrote the manuscript. All authors contributed to the article and approved the submitted version.

\section{FUNDING}

This study was supported by the National Natural Science Foundation of China (81972985).

\section{ACKNOWLEDGMENTS}

We thank the staff of the National Institute of Parasitic Disease, Chinese Center for Disease Control and Prevention for their help with parasite infections.

\section{SUPPLEMENTARY MATERIAL}

The Supplementary Material for this article can be found online at: https://www.frontiersin.org/articles/10.3389/fonc.2021.652395/ full\#supplementary-material
Cells and Modulate Innate Immunity. Nat Commun (2014) 5:5488. doi: 10.1038/ncomms6488

10. Hotez PJ, Brindley PJ, Bethony JM, King CH, Pearce EJ, Jacobson J. Helminth Infections: The Great Neglected Tropical Diseases. J Clin Invest (2008) 118 (4):1311-21. doi: 10.1172/JCI34261

11. Zhu SL, Wang S, Lin Y, Jiang PY, Cui XB, Wang XY, et al. Release of Extracellular Vesicles Containing Small RNAs From the Eggs of Schistosoma japonicum. Parasite Vector (2016) 9:574. doi: 10.1186/s13071-016-1845-2

12. He X, Wang Y, Fan X, Lei N, Tian Y, Zhang D, et al. A Schistosome miRNA Promotes Host Hepatic Fibrosis By Targeting Transforming Growth Factor Beta Receptor III. J Hepatol (2020) 72(3):519-27. doi: 10.1016/j.jhep. 2019.10.029

13. Lin Y, Zhu S, Hu C, Wang J, Jiang P, Zhu L, et al. Cross-Species Suppression of Hepatoma Cell Growth and Migration by a Schistosoma japonicum MicroRNA. Mol Ther Nucleic Acids (2019) 18:400-12. doi: 10.1016/j.omtn. 2019.09.006

14. Hu C, Zhu S, Wang J, Lin Y, Ma L, Zhu L, et al. Schistosoma japonicum MiRNA-7-5p Inhibits the Growth and Migration of Hepatoma Cells via Cross-Species Regulation of S-Phase Kinase-Associated Protein 2. Front Oncol (2019) 9:175. doi: 10.3389/fonc.2019.00175

15. Hu C, Zhu LH, Luo R, Dao JW, Zhao JP, Shi YJ, et al. Evaluation of Protective Immune Response in Mice by Vaccination the Recombinant Adenovirus for Expressing Schistosoma japonicum Inhibitor Apoptosis Protein. Parasitol Res (2014) 113(11):4261-9. doi: 10.1007/s00436-014-4104-5

16. Yin XD, Xiang TX, Li LL, Su XW, Shu XS, Luo XR, et al. DACT1, An Antagonist to Wnt/Beta-Catenin Signaling, Suppresses Tumor Cell Growth and Is Frequently Silenced in Breast Cancer. Breast Cancer Res (2013) 15(2): R23. doi: 10.1186/bcr3399 
17. Wong N, Wang XW. miRDB: An Online Resource for microRNA Target Prediction and Functional Annotations. Nucleic Acids Res (2015) 43(D1): D146-D52. doi: 10.1093/nar/gku1104

18. Kruger J, Rehmsmeier M. RNAhybrid: microRNA Target Prediction Easy, Fast and Flexible. Nucleic Acids Res (2006) 34:W451-W4. doi: $10.1093 / \mathrm{nar} / \mathrm{gkl} 243$

19. Reczko M, Maragkakis M, Alexiou P, Grosse I, Hatzigeorgiou AG. Functional microRNA Targets in Protein Coding Sequences. Bioinformatics (2012) 28 (6):771-6. doi: 10.1093/bioinformatics/bts043

20. Zhang D, Jin N, Sun W, Li X, Liu B, Xie Z, et al. Phosphoglycerate Mutase 1 Promotes Cancer Cell Migration Independent of Its Metabolic Activity. Oncogene (2017) 36(20):2900-9. doi: 10.1038/onc.2016.446

21. Hitosugi T, Zhou L, Elf S, Fan J, Kang HB, Seo JH, et al. Phosphoglycerate Mutase 1 Coordinates Glycolysis and Biosynthesis To Promote Tumor Growth. Cancer Cell (2012) 22(5):585-600. doi: 10.1016/j.ccr.2012.09.020

22. Wen YA, Zhou BW, Lv DJ, Shu FP, Song XL, Huang B, et al. Phosphoglycerate Mutase 1 Knockdown Inhibits Prostate Cancer Cell Growth, Migration, and Invasion. Asian J Androl (2018) 20(2):178-83. doi: 10.4103/aja.aja_57_17

23. Stryker ZI, Rajabi M, Davis PJ, Mousa SA. Evaluation of Angiogenesis Assays. Biomedicines (2019) 7(2):23. doi: 10.3390/biomedicines7020037

24. Bouvard V, Baan R, Straif K, Grosse Y, Secretan B, El Ghissassi F, et al. A Review of Human Carcinogens-Part B: Biological Agents. Lancet Oncol (2009) 10(4):321-2. doi: 10.1016/S1470-2045(09)70096-8

25. Humans IWGotEoCRt. Biological agents. Volume 100 B. A Review of Human Carcinogens. IARC Monogr Eval Carcinog Risks Hum (2012) 100(Pt B):1-441.

26. Chen MG. Assessment of Morbidity Due to Schistosoma japonicum Infection in China. Infect Dis Poverty (2014) 3(1):6. doi: 10.1186/2049-9957-3-6

27. Zhang R, Takahashi S, Orita S, Yoshida A, Maruyama H, Shirai T, et al. p53 Gene Mutations in Rectal Cancer Associated with Schistosomiasis japonica in Chinese Patients. Cancer Lett (1998) 131(2):215-21. doi: 10.1016/S0304-3835 (98)00154-2

28. Takemura Y, Kikuchi S, Inaba Y. Epidemiologic Study of the Relationship Between Schistosomiasis Due to Schistosoma japonicum and Liver Cancer/ Cirrhosis. Am J Trop Med Hyg (1998) 59(4):551-6. doi: 10.4269/ajtmh. 1998.59.551
29. van Tong H, Brindley PJ, Meyer CG, Velavan TP. Parasite Infection, Carcinogenesis and Human Malignancy. Ebiomedicine (2017) 15:12-23. doi: 10.1016/j.ebiom.2016.11.034

30. Ren F, Wu H, Lei Y, Zhang H, Liu R, Zhao Y, et al. Quantitative Proteomics Identification of Phosphoglycerate Mutase 1 as a Novel Therapeutic Target in Hepatocellular Carcinoma. Mol Cancer (2010) 9:81. doi: 10.1186/14764598-9-81

31. Buhrens RI, Amelung JT, Reymond MA, Beshay M. Protein Expression in Human Non-Small Cell Lung Cancer: A Systematic Database. Pathobiology (2009) 76(6):277-85. doi: 10.1159/000245893

32. Cortesi L, Barchetti A, De Matteis E, Rossi E, Della Casa L, Marcheselli L, et al. Identification of Protein Clusters Predictive of Response to Chemotherapy in Breast Cancer Patients. J Proteome Res (2009) 8(11):4916-33. doi: 10.1021/ pr900239h

33. Li C, Shu F, Lei B, Lv D, Zhang S, Mao X. Expression of PGAM1 in Renal Clear Cell Carcinoma and Its Clinical Significance. Int J Clin Exp Pathol (2015) 8(8):9410-5.

34. Potter SS. Single-Cell RNA Sequencing for the Study of Development, Physiology and Disease. Nat Rev Nephrol (2018) 14(8):479-92. doi: 10.1038/s41581-018-0021-7

35. Yan X, Xie Y, Yang F, Hua Y, Zeng T, Sun C, et al. Comprehensive Description of the Current Breast Cancer Microenvironment Advancements via SingleCell Analysis. J Exp Clin Cancer Res (2021) 40(1):142. doi: 10.1186/s13046021-01949-z

Conflict of Interest: The authors declare that the research was conducted in the absence of any commercial or financial relationships that could be construed as a potential conflict of interest.

Copyright $\odot 2021 \mathrm{Hu}, \mathrm{Li}$, Pan, Wang, Zhu, Lin, Zhu and Pan. This is an open-access article distributed under the terms of the Creative Commons Attribution License (CC BY). The use, distribution or reproduction in other forums is permitted, provided the original author(s) and the copyright owner(s) are credited and that the original publication in this journal is cited, in accordance with accepted academic practice. No use, distribution or reproduction is permitted which does not comply with these terms. 